\title{
Characterization of novel SSR markers in diverse sainfoin (Onobrychis viciifolia) germplasm
}

\author{
Katharina Kempf ${ }^{1 \dagger}$, Marina Mora-Ortiz ${ }^{2 \dagger}$, Lydia M. J. Smith, Roland Kölliker ${ }^{1}$ and Leif Skøt $^{3 *}$
}

\begin{abstract}
Background: Sainfoin is a perennial forage legume with beneficial properties for animal husbandry due to the presence of secondary metabolites. However, worldwide cultivation of sainfoin is marginal due to the lack of varieties with good agronomic performance, adapted to a broad range of environmental conditions. Little is known about the genetics of sainfoin and only few genetic markers are available to assist breeding and genetic investigations. The objective of this study was to develop a set of SSR markers useful for genetic studies in sainfoin and their characterization in diverse germplasm.
\end{abstract}

Results: A set of 400 SSR primer combinations were tested for amplification and their ability to detect polymorphisms in a set of 32 sainfoin individuals, representing distinct varieties or landraces. Alleles were scored for presence or absence and polymorphism information content of each SSR locus was calculated with an adapted formula taking into account the tetraploid character of sainfoin. Relationships among individuals were visualized using cluster and principle components analysis. Of the 400 primer combinations tested, 101 reliably detected polymorphisms among the 32 sainfoin individuals. Among the 1154 alleles amplified 250 private alleles were observed. The number of alleles per locus ranged from 2 to 24 with an average of 11.4 alleles. The average polymorphism information content reached values of 0.14 to 0.36 . The clustering of the 32 individuals suggested a separation into two groups depending on the origin of the accessions.

Conclusions: The SSR markers characterized and tested in this study provide a valuable tool to detect polymorphisms in sainfoin for future genetic studies and breeding programs. As a proof of concept, we showed that these markers can be used to separate sainfoin individuals based on their origin.

Keywords: Onobrychis viciifolia, Sainfoin, Microsatellite, SSR, Genetic diversity, Molecular markers, Fingerprinting

Abbreviations: AFLP, Amplified fragment length polymorphism; EST-SSR, Expressed sequence tag - short sequence repeats; ITS, Internal transcribed spacer; RAPD, Random amplified polymorphic DNA; SNP, Single nucleotide polymorphism; SRAP, Sequence related amplified polymorphism; SSR, Short sequence repeats

\section{Background}

Onobrychis viciifolia Scop., commonly known as sainfoin, belongs to the tribe Hedysareae and the family Fabaceae. It is a tetraploid $(2 \mathrm{n}=4 \mathrm{x}=28)$ perennial forage legume, rich in proteins and secondary plant metabolites. Its center of origin is attributed to the Middle East and Central Asia. It was introduced into Europe in

\footnotetext{
* Correspondence: Ifs@aber.ac.uk

${ }^{\dagger}$ Equal contributors

${ }^{3}$ IBERS, Aberystwyth University, Gogerddan, Aberystwyth, Ceredigion SY23 3EB, UK

Full list of author information is available at the end of the article
}

the fifteenth century and was rapidly adopted by farmers due to its high fodder value, especially for working horses [1]. Nowadays, sainfoin is cultivated only in small areas for fodder production and on ecological compensation areas. Its cultivation steadily declined since the 1950 's, due to the expanding availability of inorganic fertilizers and the preference for higher yielding legume crops such as alfalfa (Medicago sativa) or red clover (Trifolium pratense) [2-4]. In the last few years, however, sainfoin has gained renewed interest due to its animal health promoting properties associated with the 
presence of condensed tannins (CT) and other complex phytochemicals in the foliage. Benefits include anthelminthic properties and prevention of potentially lethal bloat associated with most other forage legumes [5-9]. In addition, sainfoin shows a range of beneficial agronomic features. In common with most other legumes sainfoin fixes atmospheric nitrogen in its root nodules, thus reducing the need for industrial $\mathrm{N}$ fertilizer input. $[1,10]$. Furthermore, soil fertility is improved by increased humus development through its deep rooting capability and low input requirements once established [11]. Used as a component of permanent grassland, sainfoin is a valuable alternative for areas suffering from intensification, as it increases soil fertility and has become a popular addition to non-cropped environmental planting; sainfoin provides good resources for native insects and high quality fodder for livestock [11].

Despite its advantages, a wide distribution of sainfoin is hampered by the often poor agronomic performance and the lack of sainfoin varieties adapted to different environmental conditions. The main weaknesses of sainfoin lie in its low tolerance to waterlogging and frost as well as in its poor competitive ability in the early stages of development. Therefore, targeted breeding activities are needed to select for sainfoin individuals better adapted to a broad range of environmental conditions. Breeding activities have also been impaired by the lack of knowledge of the genetic diversity of the species and its mode of inheritance. Further investigation and development of tools for marker assisted breeding has been hampered by the limited availability of species-specific molecular markers. So far, most studies have focused on the use of cross-amplifiable EST-SSRs, mainly from Medicago and Glycine species; ITS markers based on nuclear internal transcribed spacer regions and dominant SRAP markers [12-15]. The use of co-dominant SSR markers developed in other species yielded only a low number of alleles per locus in sainfoin (from 5 to 7 in bulks of 10 individual plants [12]. The development of highly informative, specific markers for sainfoin is indispensable to create a genetic knowledge base and assist breeding by marker assisted selection (MAS) [16].

SSRs or Microsatellites [17] are composed of tandemly repeated sections of DNA [18]. SSR markers show codominance of alleles and are randomly distributed along the genome, particularly in low-copy regions $[19,20]$. Considering the complex tetraploid sainfoin genome and the lack of knowledge about its genetics, SSRs are the markers of choice. SSR are multi-allelic in contrast to next generation high-throughput sequencing (NGS) derived SNP marker which are bi-allelic. This makes SSR markers highly variable and useful for distinguishing even between closely related populations or varieties [21]. Furthermore, SSR are easily detected using standard PCR methods and are transferable to related taxa [22]. The development of NGS has recently enabled the identification of a large set of set of SSR sequences from sainfoin (Mora-Ortiz et al. 2016, BMC Genomics, accepted).

In this study, our aim was to develop and characterize a comprehensive set of markers based on recently identified SSR sequences (Mora-Ortiz et al. 2016, BMC Genomics, accepted) in a panel of 32 sainfoin individuals of different origin.

\section{Methods}

\section{Plant material}

In order to include a large range of genetic diversity, we selected a set of 32 individual sainfoin plants from 29 different accessions (Table 1), originating from a range of geographical regions and showing differences for tannin content and composition [12, 13, 23, 24]. These accessions were grown in the glasshouse at the National Institute of Agricultural Botany (NIAB) (Cambridge UK) and in the field at Agroscope (Zurich, Switzerland). Young leaf material was collected from each single plant, ground in liquid nitrogen and stored at $-80^{\circ} \mathrm{C}$ until subsequent DNA extraction.

\section{DNA extraction}

DNA was extracted using the Nucleon Phytopure Genomic DNA extraction kit (GE Healthcare, Little Chalfont Buckinghamshire, United Kingdom) following the manufacturer's instructions. This method has been shown to be suitable for extraction of high quality DNA from $O$. viciifolia, in which high levels of polyphenol and condensed tannins have been reported to interfere with a successful DNA extraction using other approaches [14]. DNA quality and quantity was assessed using gel electrophoresis and spectrophotometry.

\section{PCR and Gel electrophoresis}

A total of 400 SSR primers designed from O. viciifolia transcriptome data (Mora-Ortiz 2016, unpublished), were tested with unlabeled primers for amplification in the 32 plants using an iCyler (Biorad, Hercules, USA) in a volume of $10 \mu \mathrm{L}$, with $10 \mathrm{ng}$ DNA, $1 \times$ Go Taqflexi buffer (Promega, Madison, USA), $2.5 \mathrm{mM} \mathrm{MgCl} 2$ (Promega), $0.2 \mathrm{mM}$ dNTPs (Promega), $0.2 \mu \mathrm{M}$ forward primer, $0.2 \mu \mathrm{M}$ reverse primer and $0.5 \mathrm{U}$ Polymerase G2 (Promega). The conditions followed a touchdown PCR approach with $4 \mathrm{~min}$ at $94{ }^{\circ} \mathrm{C}, 12$ cycles of $30 \mathrm{~s}$ at $66^{\circ} \mathrm{C}$ with $-1{ }^{\circ} \mathrm{C}$ decrease at each cycle plus $30 \mathrm{~s}$ at $72{ }^{\circ} \mathrm{C}$, and 30 cycles of $30 \mathrm{~s}$ at $94{ }^{\circ} \mathrm{C}, 30 \mathrm{~s}$ at $54{ }^{\circ} \mathrm{C}$ plus $30 \mathrm{~s}$ at $72{ }^{\circ}$ $\mathrm{C}$, followed by $7 \mathrm{~min}$ at $72{ }^{\circ} \mathrm{C}$. PCR products were separated by gel electrophoresis. Amplicons were separated on $1 \%$ agarose in $1 \mathrm{x}$ TBE buffer, stained with ethidium bromide and visualized under UV light. 
Table 1 O. viciifolia individuals used for marker characterization in this study

\begin{tabular}{|c|c|c|c|c|}
\hline Individual number & Variety & Status & Origin & Source \\
\hline ID_01 & 247 & NA & Morocco & GRIN \\
\hline ID_02 & Buceanskij & NA & Romania & GRIN \\
\hline ID_03 & CPI 63750 & NA & Turkey & GRIN \\
\hline ID_04 & CPI 63764 & wild & Turkey & GRIN \\
\hline ID_05 & CPI 63820 & NA & Spain & GRIN \\
\hline ID_06 & CPI 63826 & NA & Spain & GRIN \\
\hline ID_07 & NA / RCAT028437 & NA & Hungary & GRIN \\
\hline ID_08 & Ökotyp Wiedlisbach & ecotype & Switzerland & ISS \\
\hline ID_09 & Premier & landrace & Switzerland & ISS \\
\hline ID_10 & Rees A & cultivar & UK & GRIN \\
\hline ID_11 & TU86-43-03 & cultivated & Turkey & GRIN \\
\hline ID_12 & Nova & cultivar & Canada & GRIN \\
\hline ID_13 & Visnovsky & cultivar & Czech Republik & ISS \\
\hline ID_14 & Perly & cultivar & Switzerland & ISS \\
\hline ID_15 & Brunner & landrace & Austria & ISS \\
\hline ID_16 & Perdix & cultivar & Switzerland & ISS \\
\hline ID_17 & Cotswold Common & cultivar & UK & RAU \\
\hline ID_18 & Perly & cultivar & Switzerland & RAU \\
\hline ID_19 & Somborne & cultivar & UK & RAU \\
\hline ID_20 & Ibaneti/ RCAT028292 & NA & Romania & $\mathrm{RCAH}$ \\
\hline ID_21 & Bivolari/RCAT028294 & cultivar & Romania & $\mathrm{RCAH}$ \\
\hline ID_22 & NA/170582 & NA & Hungary & $\mathrm{RCAH}$ \\
\hline ID_23 & CPI 637554/ 192995 & NA & Turkey & GRIN \\
\hline ID_24 & CPI 63767 / 212241 & cultivar & USA & GRIN \\
\hline ID_25 & $\mathrm{Na} / 228352$ & wild & Iran & GRIN \\
\hline ID_26 & CPI 63781/ 236486 & NA & Turkey & GRIN \\
\hline ID_27 & Cholderton Hamshire Common & cultivar & UK & GRIN \\
\hline ID_28 & Visnovsky & cultivar & Czech Republic & GRIN \\
\hline ID_29 & Zeus & cultivar & Italy & Cotswold Seeds Ltd \\
\hline ID_30 & Zeus & cultivar & Italy & Cotswold Seeds Ltd \\
\hline ID_31 & Ambra & cultivar & Italy & private \\
\hline ID_32 & Esparcette & cultivar & UK & private \\
\hline
\end{tabular}

RAU Royal Agricultural University Gloucestershire UK, RCAH Research Centre for Agrobotany Tápiószele; Hungary, GRIN Germplasm Resources Information Network, Washington, USA, ISS Agroscope Institute for sustainability science, Zurich, Switzerland

\section{M13 PCR and capillary electrophoresis}

Those 101 primer pairs that successfully amplified fragments in the 32 individuals (Table 2) were further characterized for polymorphisms using the M13 (-21) tail primer genotyping protocol [25]. The PCR reactions were conducted in an iCycler (Biorad) in a sample volume of $10 \mu \mathrm{L}$, each containing 20 ng DNA template, 1x Go Taqflexi buffer (Promega), $1.5 \mathrm{mM} \mathrm{MgCl}_{2}$, (Promega), $0.2 \mathrm{mM}$ dNTPs (Promega), $0.16 \mu \mathrm{M}$ forward primer carrying the M13-tail, $0.04 \mu \mathrm{M}$ reverse primer and $0.16 \mu \mathrm{M}$ fluorescently labelled M13-primer, $0.5 \mathrm{U}$ polymerase GoTaq G2 (Promega).
PCR conditions were $2 \mathrm{~min}$ at $94^{\circ} \mathrm{C}, 30$ cycles of $30 \mathrm{~s}$ at $94{ }^{\circ} \mathrm{C}, 45 \mathrm{~s}$ at $56{ }^{\circ} \mathrm{C}$ and $45 \mathrm{~s}$ at $72{ }^{\circ} \mathrm{C}$, followed by 8 cycles of $30 \mathrm{~s}$ at $94{ }^{\circ} \mathrm{C}, 45 \mathrm{~s}$ at $53{ }^{\circ} \mathrm{C}$ and $45 \mathrm{~s}$ at $72{ }^{\circ} \mathrm{C}$. The final extension step was conducted at $72{ }^{\circ} \mathrm{C}$ for 10 min. An aliquot of $1 \mu \mathrm{l}$ of the PCR product was diluted in $10 \mu \mathrm{HiDi}^{\mathrm{rm}}$ formamide (Applied Biosystems ${ }^{\oplus}$, Thermo Fisher Scientific, Waltham, MA, USA) and $0.2 \mu \mathrm{l}$ Rox $500^{\mathrm{Tm}}$ oligonucleotide 'size ladder'(Applied Biosystems ${ }^{\oplus}$ ) for capillary electrophoresis on the Genetic Analyzer 3730 (Life Technologies, Carlsbad, CA). Alleles were scored using the GeneMarker software (Softgenetics, V2.4.0 Inc., State College, USA). 
Table 2 SSR primer sequences used for amplification in 32 O. viciifolia individuals and characteristics of SSR motifs

\begin{tabular}{|c|c|c|c|c|c|}
\hline Marker & Motif & Repeats & Predicted size & Forward primer $\left(5^{\prime}-3^{\prime}\right)$ & Reverse primer $\left(5^{\prime}-3^{\prime}\right)$ \\
\hline OVK002 & $A G$ & 9 & 164 & CCCACCAGACAAAAAGAATA & GCTTTCCCCTTCATCAACTAT \\
\hline OVK003 & TA & 8 & 122 & GATAGAATTCGTITGTTGGTG & ATCTTTGTAACTGTTCGCTCA \\
\hline OVK017 & $A C$ & 8 & 158 & GGGTGTTAGTTATCCATTTCC & ACATACTAGCCTTCTGGGGTA \\
\hline OVK027 & CTCG & 6 & 129 & AATGGAATCTCGGAGACAG & GGAAGAAGACGAAGTAGTAGGA \\
\hline OVK034 & $\mathrm{GCT}$ & 6 & 150 & GTGAGATGAGCTTGGACATT & AGATAACTAACTGCAGGCAAG \\
\hline OVK036 & AGGT & 6 & 150 & GTGTTAAAGGGGTGAAAACAT & CATTTTGACAAACCAGTATCC \\
\hline OVK038 & ATT & 6 & 166 & CCACATACGAGACAGAATAGG & CTGAAAATTGATCGATACTGG \\
\hline OVK042 & GTT & 6 & 144 & GGAACGGTTAATTTCTGATIT & AGAATTCCGTACAAGTCGAG \\
\hline OVK045 & AGA & 6 & 148 & CCAAAAATCATCAATCAACAC & TTGAACAAGGGTTAGGGTTAT \\
\hline OVK046 & AGTG & 6 & 151 & TCAACCACATTATAAAACCTCA & CGCGAAATCATAGTTCACTT \\
\hline OVK054 & GAA & 6 & 201 & TTGCAGAGATAACACTCACCT & TCCTGAAAAACCTAATCACAA \\
\hline OVK055 & GAT & 6 & 189 & GAAGATATTTCAAAGCAGCAA & CATGCTACCACTAGCAGAAGT \\
\hline OVK063 & TTG & 6 & 188 & AATTGCAACTGAAACTGAAAC & ACTGCTACCCTCTCCATAAAT \\
\hline OVK068 & GGA & 6 & 195 & GACCACCCGCAGCTCAAC & GTCTTCTTCCCCCATATTTAG \\
\hline OVK072 & ACC & 7 & 199 & TTGCCTTAGTCAGTTACCTTG & GTGGAGAGAATGAGAGAACCT \\
\hline OVK073 & GAC & 6 & 200 & GTAGACAACCGTATCTGGACA & AAGATGGAAGGTTCTAGTTCG \\
\hline OVK077 & TTA & 6 & 249 & GTCCСTCTCTCTCAAATTGTT & AGGTTAATGGAGCTTAGTGGT \\
\hline OVK089 & CAT & 6 & 257 & CAAAGTCATACCAATCACCAT & TCTTGGAAGCACTTGTTACTC \\
\hline OVK093 & CCA & 6 & 259 & CCAAGTGTTGAAAGTCTCAG & TGAGAGTTCGTTCAAGGTAGA \\
\hline OVK094 & TTGCG & 5 & 255 & ACCGATCTTAGGATAGATGGA & ACTTTTGGTTGCTTAGTCGAT \\
\hline OVK096 & TCA & 6 & 249 & GAGCGTTGCATTTACATTTAC & САTCСТССТTТАСАCССТАAT \\
\hline OVK097 & GTGA & 6 & 252 & TCTATAGAGATGAGGCGACAA & CGCCCCTAACTAACCTACTAC \\
\hline OVK099 & TGAG & 6 & 247 & AGAAAATGGAAGCAACAGAGT & ACAAATAGCAGCTCCCTTC \\
\hline OVK101 & CTAA & 6 & 254 & GTTGAGTTCAGACACAGAGC & AATAGCTCCCACAATAACTCC \\
\hline OVK102 & TGT & 6 & 249 & CCAAAGGGTGTITATITTCT & GGAAGAAATTAAGCAAATGGT \\
\hline OVK107 & $A G$ & 8 & 193 & AAGTTAAAACTTTGCGTTGTG & GACGTTGTTCTGGATTTCTTC \\
\hline OVK111 & GGT & 7 & 206 & TATAGACCTTCTCCTCCCAGA & GTGAAAGTCACAAATCCAAAG \\
\hline OVK119 & CAG & 6 & 199 & АСССТССТТСТСТССТTATTा & GACGAGAGAACTCGTTTATGA \\
\hline OVK122 & $\mathrm{TC}$ & 9 & 211 & GCAGATAGCACAGTTATCGAC & GAACCACACACACAGAATCA \\
\hline OVK123 & ACA & 9 & 200 & CACCCATTAACTATCATGGTC & CAAGCCCTITGTGAGATACTA \\
\hline OVK124 & TGA & 9 & 211 & GCCTITTCTGTGACTCGTAA & GCTCCATTCCCATTTATAGTT \\
\hline OVK125 & CATTT & 5 & 193 & AAATTTAAGCACCGGAATAAC & AAAGCAAAAGGGCTACTAAAG \\
\hline OVK126 & $\mathrm{TC}$ & 8 & 197 & CGACAAAACTATTTAGGCAAA & GGGAAGAGATCATAAACCCTA \\
\hline OVK127 & AT & 8 & 200 & GCCCAAAATGTATTATCCTTC & AGAACAGACAGATATGCAAGC \\
\hline OVK131 & TA & 8 & 200 & TCTATCTGGGTGTTGTITGT & CTGTTTGAATATCGATTACCAC \\
\hline OVK133 & $\mathrm{TG}$ & 8 & 196 & TGCTTCAGCATTATTGTAACAT & TGCACTTCTCCATACTTCCTA \\
\hline OVK138 & CTAA & 6 & 250 & TAATATGGTGCAAGTTCCAAT & TTCTACGCTTAGCTCAAACC \\
\hline OVK141 & CACG & 6 & 239 & GAGGAGGTACATACAGCACAG & CAACCTCCTCGTTATCTITTT \\
\hline OVK142 & GT & 8 & 243 & AACATGACTACTGTGAACAAGG & CGAACATGTAATTGATCCAAG \\
\hline OVK155 & GTG & 6 & 251 & CAGGTTGAAGTAGCAGAGAA & GTAGACCACGCATACTGAATC \\
\hline OVK158 & GACT & 6 & 257 & TCAGAGTGTGTTGTGTTGTGT & AGTGAAGCAAATGTGTGATTT \\
\hline OVK159 & $\mathrm{TG}$ & 8 & 251 & CATTATTGCCTAGCATTGTTC & ATTTCACCATCAAGTATGCAC \\
\hline OVK161 & TTCC & 6 & 249 & AAAGCTTTCTACACGTTGGTA & TGGGTTITTACACTCTGTGAT \\
\hline OVK165 & ACA & 6 & 267 & ТTCAAACACTCACTCACTCC & TCGGATTTGTGACCTAACTC \\
\hline
\end{tabular}


Table 2 SSR primer sequences used for amplification in 32 O. viciifolia individuals and characteristics of SSR motifs (Continued)

\begin{tabular}{|c|c|c|c|c|c|}
\hline OVK168 & TGA & 6 & 253 & AATTATCACCCACTGCTATGA & GGTTTCCATCACTGTTTGTA \\
\hline OVK172 & AGC & 6 & 256 & TTATTAAACCTGCGTCTTCTG & GTAGAGCTGTGGGCTITATCT \\
\hline OVK173 & $C T$ & 8 & 253 & TCGTTCTCGTGATTATTCTGT & ССTCTATTCAAATAGGGCAAT \\
\hline OVK174 & GGCCC & 5 & 246 & ACATGATCGTGAATATGAAGC & CAGCAGCAATCAATATATCATC \\
\hline OVK175 & CA & 9 & 250 & GTAAAATATCAAGCAGGAGCA & AAACTATGCAGACACCCTGTA \\
\hline OVK177 & $\mathrm{CTG}$ & 7 & 257 & TCTGTTGATTTAAGGAGACGA & СTCTTGCTCATATTTTCCTCA \\
\hline OVK181 & AAG & 6 & 257 & AGGAAGAAGAAGAAGAAGCAG & TTCTCCTITAACCACAACCTT \\
\hline OVK183 & TGAT & 6 & 256 & GAGGGTAAGAGAGAGTGGAAG & CTTGCCTGATATCTTCTCAAA \\
\hline OVK196 & AGC & 6 & 286 & TाTGAGAGTGTGGAAGGTTA & AGTATGAGCCTGATGATGATG \\
\hline OVM003 & $\mathrm{TC}$ & 9 & 297 & CCGTCTGTTTAATCATTCACT & GAAAGGAAAGGTTATTGGAGA \\
\hline OVM004 & ATाT & 5 & 290 & GGGAATTCTTAAATCTCATGG & ATGCATGGTACTGGGTCGT \\
\hline OVM025 & CAA & 6 & 297 & TTCTGAACAACAACAACAACA & GTCCAGGAGCTAAGTAACCAT \\
\hline OVM031 & TGA & 6 & 306 & ATTGGTTTCTAAGGAGGACTG & GCAATACTCCTCTGCCTAGTT \\
\hline OVM033 & CTC & 6 & 300 & CAAGGCTTATTTGGTTAACAG & ATACTATTTCCCATGCCTACC \\
\hline OVM034 & TTC & 6 & 308 & GCATTTCATCAAACACTITTC & TTGGTTGAATCTGTGAGACT \\
\hline OVM035 & TTC & 7 & 303 & TCATCAAACACTITCGTTCT & TTGGTTGAATCTGTGAGACT \\
\hline OVM038 & GAAG & 6 & 297 & CACAGGACAAGAGTGAGAGAG & TCATGATACCACGAATITITC \\
\hline OVM043 & GAG & 7 & 167 & TAGTATGGCTGAAATCAAAGG & ATATCATAAGGGCAACAGTGA \\
\hline OVM048 & AT & 9 & 157 & GACATTGAAATCAAACAATCC & AACACTTGTCATGTITCCAAG \\
\hline OVM049 & TGA & 7 & 150 & AACAAACAAGAGGAAAAGGAG & TATGTGCTTATCAGGCATTTT \\
\hline OVM050 & ATCC & 6 & 161 & ATGAGCATGAAGAGTTCAGA & ACACATCTACGACTTCTITCG \\
\hline OVM053 & GTGGA & 5 & 149 & CACCAAAAGCATAGCAATAGT & GCTTGAATTGAATGAGAAATG \\
\hline OVM057 & TTG & 6 & 153 & CCTTGAGGAGGAATAATAGGA & GACATCATCATCACCTTCACT \\
\hline OVM058 & AT & 9 & 150 & GTCAAGTCATACCCATACGAG & CAGTGTAACCATATGCACAAA \\
\hline OVM059 & AGA & 6 & 149 & ACTCCAACTCCAACTCAGAAC & AAGCGAAGAAGAGAGTGAGAT \\
\hline OVM060 & $C T$ & 8 & 159 & ATGTAATCAAAAGGTGCAGAA & AGCTTCCAAAACAGTGTATGA \\
\hline OVM061 & GTA & 6 & 150 & TTAACACACGTACGTACCACA & TTTGTCGTTGATCGTTAAGTT \\
\hline OVM062 & $A G$ & 8 & 139 & GGAAAAAGGTTTGGATAGATG & AAGTTTTCCCCACACTATTCT \\
\hline OVM064 & AT & 8 & 353 & GCATGCACAGAATTAAGTTC & AGAAGGTCCTITGAAAATCAG \\
\hline OVM065 & $\mathrm{CT}$ & 8 & 352 & AAGACAGCGAGTTACCAATCT & GATTGAAACTGAGTAGCGATG \\
\hline OVM067 & $\mathrm{CTT}$ & 6 & 352 & CAACCTTAATACCAACCTTCC & AAAAGTAGCCAGAGAGCAAAT \\
\hline OVM068 & $\mathrm{CCT}$ & 6 & 333 & CTACAACTCACCGAAACTCAC & CGATTTCTGCCTCTTTATTCT \\
\hline OVM069 & AATG & 6 & 357 & ATGTTGTACAGATGAGCTTCG & TAGTGAGCAAACCTATITGG \\
\hline OVM072 & GAA & 6 & 350 & TTGATGTGGTTGATCCTATTC & GATGTCAACATCTTGGTCATTA \\
\hline OVM073 & ACA & 6 & 346 & GTTCTCAAACGCACTATCAAC & AAAATCTTGTAGGGATTCGAT \\
\hline OVM076 & AAC & 7 & 348 & СССАТTСТTСАТСТTСТTСТC & TGCTTCCATAATCAGTGAAAT \\
\hline OVM081 & GT & 9 & 350 & TCTAGCACAATGTTTTGGATT & TATTGAGTTGAAGCAGACCAT \\
\hline OVM083 & $C T$ & 8 & 347 & CACACAAACACAAAACTCACA & GATCGGAGAAAAGAAGAAGAG \\
\hline OVM086 & GAA & 6 & 350 & TCATACAAAGTTCCTTCCGTA & ATTGCCAATAACAGTGAAGAG \\
\hline OVM090 & CCA & 6 & 151 & AATCAATGGAGGAGGATAAAC & GAAGGTTGAAAAGGGAATAAA \\
\hline OVM091 & ATC & 6 & 188 & AACCACCCTTAATTCCATAAG & AGATAAAAGCCGCAAAAGTAT \\
\hline OVM092 & CAC & 6 & 157 & GGACCAACAAAGAGGATTATT & CCCTTGCTTGAAGTGTTACTA \\
\hline OVM094 & $\mathrm{GTT}$ & 5 & 163 & ATTCATGGGGACAATAAATTC & CAAGAGAATGAATGAATCAGC \\
\hline OVM099 & GA & 9 & 149 & TATGTATTGCAGAATCACAGC & TATTACCCTTTTCCATCTTCC \\
\hline OVM100 & AAG & 6 & 151 & GAACTAGATTTGCGGCATT & СССАСАСССТТАТССТТАТTА \\
\hline
\end{tabular}


Table 2 SSR primer sequences used for amplification in 32 O. viciifolia individuals and characteristics of SSR motifs (Continued)

\begin{tabular}{llllll}
\hline OVM110 & AT & 8 & 154 & CTGGACGAAAACAACATATTC & GTTGGCTTGGTACTGACATA \\
OVM116 & GAT & 7 & 151 & AACTACACGCACGTAATGAAT & TGGTTGATAAACACCTCAAG \\
OVM120 & TTC & 6 & 152 & TTCAGTGTCACTTCCTCATT & AGAAGTTGTCATGTCAAGGAA \\
OVM122 & TGG & 6 & 156 & ATGAATCTTGTACGGAATCTG & GAAGAAAAAGCCATAAACACC \\
OVM125 & AAATT & 5 & 151 & ATTCTTCAACAAGCAAGTGA & CTGCAATTCCATCCTATTTA \\
OVM126 & TCC & 6 & 188 & ACTAAGAACCACCCAAAACAT & TGAGAAGATGGAGAAGATGTG \\
OVM128 & TGT & 6 & 155 & GAGAAGCATAACCAAAATCCT & TGGAAGAAAAGAAACTTCTGA \\
OVM129 & TG & 8 & 133 & AATTGGATTCATGTGTAGGA & GAAGTGGAGCCAAAACCT \\
OVM130 & AG & 9 & 154 & GCAAATTATCACCATGCAC & CGTGAAGAAAATCGGTACTTA \\
OVM131 & AGA & 6 & 153 & ACGGTAATAACGCAGGCAGATAC & AATTAGAGGCTTCGACTTGTT \\
OVM132 & GAC & 6 & 142 & TAGCATCAAGGTTGGAAATAG & CTAGGCTACCTGAATCAAACA \\
OVM133 & TITC & 5 & 171 & &
\end{tabular}

\section{Data analysis}

All statistical analyses and calculations were performed using R statistical software ( $\mathrm{R}$ Core Team, 2014). The polymorphism information content (PIC) of SSR markers was calculated as the mean of the PIC of each allele, using the formula for dominant markers from Roldan-Ruiz et al. [26] as;

$$
P I C_{i}=2 f_{i}\left(1-f_{i}\right)
$$

where $\mathrm{PIC}_{\mathrm{i}}$ is the polymorphism information content of allele $i$ and $f_{i}$ is the frequency of occurrence of allele $i$ (fragment present) in the 32 individuals. From single alleles, average $\left(\mathrm{PIC}_{\mathrm{Av}}\right)$, minimum $\left(\mathrm{PIC}_{\mathrm{Min}}\right)$ and maximum $\left(\mathrm{PIC}_{\mathrm{Max}}\right)$ PIC values were calculated for each SSR marker.

In order to calculate genetic distance measures, SSR alleles were coded as individual markers with 1 for presence and 0 for absence of the allele as binary data. Pairwise genetic distances between individuals were calculated as modified Rogers' distance $D_{w}$, [27] which shows the extent of genetic diversity between two individuals [28] ranging from 0 (no diversity between individuals) and 1 (maximum diversity).

Genetic relationships were visualised using cluster analysis and the R-function pvclust() [29] based on Euclidean distance that was rescaled to $D_{w}$ for plotting purposes $\left(D_{w}\right.$ and Euclidean distance show a linear relationship, Additional file 1: Figure S1). Probability values ( $p$-values) were calculated for each cluster using multiscale bootstrap resampling $[30,31]$ to calculate approximately unbiased (AU) $p$-values [32]. The k-means clustering algorithm [33] was applied to the $D_{w}$ values using a sequence of $k=2$ clusters to 32 clusters. The Calinsky criterion [34] was then calculated for each number of $\mathrm{k}$ as implemented in the $\mathrm{R}$ function cascadeKM() and the optimum number of clusters was determined at the maximal value. Population structure was further investigated by principal component analysis performed on binary raw data of individual alleles.

\section{Results}

SSR analysis

SSR markers showed a high degree of polymorphism and overall, 1154 alleles were found with an average of 11.4 alleles per marker locus (Table 3). Among those 1154 alleles, only five alleles (from SSR OVK042, OVK172, OVM031, OVM072 and OVM100) were nonpolymorphic and hence present in all individuals studied.

With only two alleles in the 32 individuals, SSR OVK042 had the lowest number of alleles, whereas OVK158 had the highest number with 24 amplified alleles. The minimum rate of allele occurrence was 0.03125 , corresponding to occurrence in only one genotype (i.e. a private allele of an individual genotype). In total, 250 private alleles were detected and these were equally distributed across the examined set of individuals and markers. With regard to individuals, the highest number of private alleles over all markers was found for individual ID_08 (14 private alleles) and the lowest number was found for ID_17 (3 private alleles). The origin of the individual did not appear to affect the occurrence of private alleles. With regard to markers, the most private alleles were observed in OVM064 (8 private alleles), whereas 16 markers $(15.8 \%)$ had no private alleles at all.

The average polymorphism information content $\left(\mathrm{PIC}_{\mathrm{Av}}\right)$ ranged from 0.14 (OVK141) to 0.36 (OVK101) (Table 3). A detailed look at the PIC values of individual alleles in the different markers exhibited minimum PIC values per SSR $\left(\mathrm{PIC}_{\mathrm{Min}}\right)$ between 0 (Additional file 2: Figure S2), OVK042, OVK172, OVM031, OVM072, OVM100) and 0.17 (OVK131) and maximum PIC values per SSR (PICMax) between 0.3 (OVK 172) and 0.5 (16 different markers). 
Table 3 Characterization of the 101 polymorphic sainfoin markers

\begin{tabular}{|c|c|c|c|c|c|c|c|c|}
\hline Marker & PIC Av & PIC Min & PIC Max & $\mathrm{NoA}$ & NoA Priv & MinAF & MaxAF & Size \\
\hline OVKO02 & 0.22 & 0.06 & 0.47 & 9 & 3 & 0.03 & 0.63 & $154-175$ \\
\hline OVK003 & 0.23 & 0.06 & 0.47 & 11 & 2 & 0.03 & 0.38 & $92-124$ \\
\hline OVK017 & 0.22 & 0.06 & 0.50 & 19 & 5 & 0.03 & 0.47 & 148-184 \\
\hline OVK027 & 0.28 & 0.06 & 0.50 & 9 & 2 & 0.03 & 0.59 & $120-140$ \\
\hline OVK034 & 0.27 & 0.06 & 0.49 & 12 & 1 & 0.03 & 0.56 & 138-154 \\
\hline OVK036 & 0.35 & 0.17 & 0.50 & 7 & 0 & 0.09 & 0.69 & 133-154 \\
\hline OVK038 & 0.19 & 0.06 & 0.40 & 14 & 4 & 0.03 & 0.28 & $155-186$ \\
\hline OVK042 & 0.25 & 0.00 & 0.50 & 2 & 0 & 0.50 & 1.00 & 183-186 \\
\hline OVK045 & 0.29 & 0.12 & 0.43 & 6 & 0 & 0.09 & 0.94 & $138-148$ \\
\hline OVK046 & 0.31 & 0.06 & 0.49 & 12 & 1 & 0.03 & 0.56 & $138-157$ \\
\hline OVK054 & 0.29 & 0.12 & 0.49 & 15 & 0 & 0.06 & 0.44 & 274-290 \\
\hline OVK055 & 0.20 & 0.06 & 0.38 & 8 & 2 & 0.03 & 0.84 & 135-159 \\
\hline OVK063 & 0.24 & 0.06 & 0.50 & 13 & 2 & 0.03 & 0.72 & $179-200$ \\
\hline OVK068 & 0.25 & 0.06 & 0.43 & 9 & 3 & 0.03 & 0.31 & $186-213$ \\
\hline OVK072 & 0.32 & 0.12 & 0.50 & 4 & 0 & 0.06 & 0.81 & 193-198 \\
\hline OVK073 & 0.29 & 0.06 & 0.50 & 11 & 1 & 0.03 & 0.53 & $186-210$ \\
\hline OVK077 & 0.23 & 0.06 & 0.45 & 9 & 2 & 0.03 & 0.78 & 233-264 \\
\hline OVK089 & 0.27 & 0.06 & 0.49 & 9 & 2 & 0.03 & 0.44 & 279-299 \\
\hline OVK093 & 0.23 & 0.06 & 0.50 & 14 & 6 & 0.03 & 0.56 & 234-271 \\
\hline OVK094 & 0.24 & 0.06 & 0.48 & 14 & 4 & 0.03 & 0.66 & 208-244 \\
\hline OVK096 & 0.21 & 0.06 & 0.48 & 20 & 6 & 0.03 & 0.41 & 215-294 \\
\hline OVK097 & 0.22 & 0.06 & 0.38 & 3 & 0 & 0.13 & 0.97 & $240-248$ \\
\hline OVK099 & 0.25 & 0.06 & 0.49 & 13 & 2 & 0.03 & 0.75 & $232-270$ \\
\hline OVK101 & 0.36 & 0.06 & 0.50 & 7 & 1 & 0.03 & 0.72 & 339-352 \\
\hline OVK102 & 0.23 & 0.06 & 0.34 & 4 & 1 & 0.03 & 0.22 & 239-251 \\
\hline OVK107 & 0.29 & 0.06 & 0.45 & 15 & 1 & 0.03 & 0.72 & 206-234 \\
\hline OVK111 & 0.26 & 0.06 & 0.48 & 7 & 2 & 0.03 & 0.75 & 213-232 \\
\hline OVK119 & 0.30 & 0.06 & 0.47 & 10 & 1 & 0.03 & 0.72 & 216-252 \\
\hline OVK122 & 0.24 & 0.06 & 0.45 & 8 & 1 & 0.03 & 0.66 & $330-341$ \\
\hline OVK123 & 0.26 & 0.06 & 0.50 & 10 & 3 & 0.03 & 0.75 & 208-237 \\
\hline OVK124 & 0.26 & 0.06 & 0.49 & 15 & 1 & 0.03 & 0.44 & 218-267 \\
\hline OVK125 & 0.29 & 0.06 & 0.50 & 9 & 1 & 0.03 & 0.72 & $197-222$ \\
\hline OVK126 & 0.25 & 0.06 & 0.49 & 15 & 3 & 0.03 & 0.56 & 198-233 \\
\hline OVK127 & 0.28 & 0.06 & 0.49 & 6 & 1 & 0.03 & 0.44 & 204-222 \\
\hline OVK131 & 0.17 & 0.06 & 0.48 & 15 & 3 & 0.03 & 0.59 & $183-228$ \\
\hline OVK133 & 0.25 & 0.06 & 0.50 & 13 & 3 & 0.03 & 0.63 & $205-239$ \\
\hline OVK138 & 0.21 & 0.06 & 0.49 & 13 & 6 & 0.03 & 0.56 & $232-267$ \\
\hline OVK141 & 0.14 & 0.06 & 0.47 & 15 & 7 & 0.03 & 0.38 & $242-269$ \\
\hline OVK142 & 0.25 & 0.06 & 0.50 & 12 & 3 & 0.03 & 0.47 & 256-285 \\
\hline OVK155 & 0.24 & 0.06 & 0.49 & 14 & 3 & 0.03 & 0.56 & 234-282 \\
\hline OVK158 & 0.19 & 0.06 & 0.40 & 24 & 6 & 0.03 & 0.28 & $273-375$ \\
\hline OVK159 & 0.23 & 0.06 & 0.50 & 14 & 4 & 0.03 & 0.81 & $268-290$ \\
\hline OVK161 & 0.25 & 0.06 & 0.40 & 12 & 1 & 0.03 & 0.28 & $220-276$ \\
\hline OVK165 & 0.19 & 0.06 & 0.50 & 20 & 6 & 0.03 & 0.50 & $273-311$ \\
\hline
\end{tabular}


Table 3 Characterization of the 101 polymorphic sainfoin markers (Continued)

\begin{tabular}{|c|c|c|c|c|c|c|c|c|}
\hline OVK168 & 0.24 & 0.06 & 0.50 & 11 & 3 & 0.03 & 0.81 & $258-284$ \\
\hline OVK172 & 0.16 & 0.00 & 0.30 & 5 & 0 & 0.06 & 1.00 & $268-279$ \\
\hline OVK173 & 0.23 & 0.06 & 0.48 & 18 & 5 & 0.03 & 0.59 & $268-316$ \\
\hline OVK174 & 0.23 & 0.06 & 0.48 & 5 & 2 & 0.03 & 0.75 & $245-266$ \\
\hline OVK175 & 0.19 & 0.06 & 0.38 & 10 & 2 & 0.03 & 0.88 & $252-267$ \\
\hline OVK177 & 0.27 & 0.06 & 0.49 & 7 & 2 & 0.03 & 0.59 & $267-286$ \\
\hline OVK181 & 0.19 & 0.06 & 0.38 & 19 & 4 & 0.03 & 0.25 & $343-381$ \\
\hline OVK183 & 0.24 & 0.06 & 0.49 & 17 & 1 & 0.03 & 0.44 & $266-289$ \\
\hline OVK196 & 0.21 & 0.06 & 0.50 & 8 & 2 & 0.03 & 0.53 & $297-314$ \\
\hline OVM003 & 0.31 & 0.12 & 0.47 & 10 & 0 & 0.06 & 0.69 & 299-321 \\
\hline OVM004 & 0.21 & 0.06 & 0.45 & 18 & 6 & 0.03 & 0.34 & $380-426$ \\
\hline OVM025 & 0.33 & 0.17 & 0.49 & 7 & 0 & 0.09 & 0.84 & $306-324$ \\
\hline OVM031 & 0.26 & 0.00 & 0.50 & 13 & 1 & 0.03 & 1.00 & $292-353$ \\
\hline OVM033 & 0.29 & 0.06 & 0.50 & 8 & 1 & 0.03 & 0.69 & $308-330$ \\
\hline OVM034 & 0.22 & 0.06 & 0.50 & 17 & 5 & 0.03 & 0.53 & $307-355$ \\
\hline OVM035 & 0.22 & 0.06 & 0.50 & 17 & 5 & 0.03 & 0.53 & $301-350$ \\
\hline OVM038 & 0.19 & 0.06 & 0.43 & 14 & 4 & 0.03 & 0.31 & $311-351$ \\
\hline OVM043 & 0.30 & 0.06 & 0.49 & 10 & 2 & 0.03 & 0.66 & 173-203 \\
\hline OVM048 & 0.29 & 0.12 & 0.43 & 6 & 0 & 0.06 & 0.72 & 174-186 \\
\hline OVM049 & 0.31 & 0.06 & 0.50 & 9 & 1 & 0.03 & 0.50 & $162-198$ \\
\hline OVM050 & 0.20 & 0.06 & 0.49 & 13 & 6 & 0.03 & 0.72 & 168-198 \\
\hline OVM053 & 0.32 & 0.06 & 0.50 & 11 & 1 & 0.03 & 0.50 & 134-182 \\
\hline OVM057 & 0.35 & 0.17 & 0.49 & 5 & 0 & 0.09 & 0.66 & $165-180$ \\
\hline OVM058 & 0.23 & 0.06 & 0.49 & 15 & 3 & 0.03 & 0.44 & $135-178$ \\
\hline OVM059 & 0.24 & 0.06 & 0.48 & 7 & 2 & 0.03 & 0.59 & $156-174$ \\
\hline OVM060 & 0.23 & 0.06 & 0.50 & 21 & 4 & 0.03 & 0.50 & $172-219$ \\
\hline OVM061 & 0.19 & 0.06 & 0.50 & 10 & 4 & 0.03 & 0.84 & $143-175$ \\
\hline OVM062 & 0.30 & 0.06 & 0.49 & 12 & 2 & 0.03 & 0.59 & $151-170$ \\
\hline OVM064 & 0.16 & 0.06 & 0.47 & 16 & 8 & 0.03 & 0.38 & $380-444$ \\
\hline OVM065 & 0.25 & 0.06 & 0.49 & 14 & 4 & 0.03 & 0.69 & $360-391$ \\
\hline OVM067 & 0.33 & 0.17 & 0.49 & 6 & 0 & 0.09 & 0.66 & $366-380$ \\
\hline OVM068 & 0.26 & 0.12 & 0.43 & 8 & 0 & 0.06 & 0.88 & $343-368$ \\
\hline OVM069 & 0.26 & 0.06 & 0.48 & 13 & 2 & 0.03 & 0.59 & $454-479$ \\
\hline OVM072 & 0.28 & 0.00 & 0.50 & 7 & 1 & 0.03 & 1.00 & $365-387$ \\
\hline OVM073 & 0.20 & 0.06 & 0.45 & 21 & 5 & 0.03 & 0.34 & $446-511$ \\
\hline OVM076 & 0.22 & 0.06 & 0.48 & 17 & 3 & 0.03 & 0.41 & $347-376$ \\
\hline OVM081 & 0.18 & 0.06 & 0.40 & 17 & 5 & 0.03 & 0.28 & 353-396 \\
\hline OVM083 & 0.30 & 0.06 & 0.50 & 11 & 2 & 0.03 & 0.63 & $365-384$ \\
\hline OVM086 & 0.32 & 0.06 & 0.50 & 10 & 1 & 0.03 & 0.63 & $371-391$ \\
\hline OVM090 & 0.30 & 0.06 & 0.49 & 8 & 2 & 0.03 & 0.84 & $158-180$ \\
\hline OVM091 & 0.34 & 0.06 & 0.50 & 8 & 1 & 0.03 & 0.47 & $184-217$ \\
\hline OVM092 & 0.23 & 0.06 & 0.43 & 7 & 2 & 0.03 & 0.81 & 163-185 \\
\hline OVM094 & 0.33 & 0.12 & 0.50 & 7 & 0 & 0.06 & 0.81 & 190-207 \\
\hline OVM099 & 0.18 & 0.06 & 0.50 & 11 & 5 & 0.03 & 0.50 & $165-198$ \\
\hline OVM100 & 0.24 & 0.00 & 0.48 & 5 & 0 & 0.09 & 1.00 & $163-179$ \\
\hline
\end{tabular}


Table 3 Characterization of the 101 polymorphic sainfoin markers (Continued)

\begin{tabular}{|c|c|c|c|c|c|c|c|c|}
\hline OVM110 & 0.21 & 0.06 & 0.49 & 18 & 5 & 0.03 & 0.44 & 163-185 \\
\hline OVM116 & 0.28 & 0.06 & 0.49 & 15 & 2 & 0.03 & 0.56 & 138-204 \\
\hline OVM120 & 0.34 & 0.06 & 0.50 & 6 & 1 & 0.03 & 0.88 & 169-187 \\
\hline OVM122 & 0.31 & 0.06 & 0.50 & 4 & 1 & 0.03 & 0.91 & 164-180 \\
\hline OVM125 & 0.26 & 0.06 & 0.50 & 10 & 3 & 0.03 & 0.47 & $161-180$ \\
\hline OVM126 & 0.22 & 0.06 & 0.50 & 18 & 4 & 0.03 & 0.53 & $191-229$ \\
\hline OVM128 & 0.26 & 0.06 & 0.47 & 9 & 1 & 0.03 & 0.81 & 173-190 \\
\hline OVM129 & 0.25 & 0.06 & 0.49 & 14 & 4 & 0.03 & 0.56 & $146-173$ \\
\hline OVM130 & 0.20 & 0.06 & 0.47 & 20 & 7 & 0.03 & 0.38 & 152-187 \\
\hline OVM131 & 0.34 & 0.06 & 0.50 & 8 & 2 & 0.03 & 0.56 & 159-198 \\
\hline OVM132 & 0.30 & 0.06 & 0.47 & 8 & 2 & 0.03 & 0.78 & $157-176$ \\
\hline OVM133 & 0.20 & 0.06 & 0.47 & 14 & 3 & 0.03 & 0.63 & $177-212$ \\
\hline
\end{tabular}

PICAv, PICMin and PICMax give the average, minimum and maximum allele-wise polymorphism information content values, NoA $\mathrm{Tot}_{\mathrm{T}}$ the total number of alleles, $\mathrm{NoA}_{\text {Priv }}$ the number of private alleles, MinAF the minimum allele frequency and MaxAF the maximum allele frequency value

The overall length of SSR fragments detected ranged from 91 to 511base pairs (bp). Markers with two base pair motifs had a slightly higher number of repeats (eight to nine) when compared to markers with three to five bp motifs (five to seven repetitions). The total fragment length observed did not differ between motif lengths (data not shown). Contrastingly, the number of alleles found for SSRs with two bp motifs was higher (13.5 alleles on average), compared to SSRs with longer motifs (10.7 alleles). The average number of alleles per sainfoin genotype was 230.1 over all SSR markers, leading to an average of 2.3 alleles per SSR marker and genotype. The lowest number of alleles was found for genotype ID_25 with 191 alleles, the highest for ID_07 with 268 alleles. Assigning all individuals to cultivars and non-cultivars (ecotypes, landraces and NA) resulted in 981 alleles for individuals from cultivars (57.7 alleles per individual) and 942 alleles for non-cultivars (62.8 alleles per individual).

\section{Diversity of $O$. viciifolia individuals}

The allocation of individuals to groups by overall similarity of alleles was assessed using k-means partition comparisons. Those k-means statistic (Fig. 1, left)

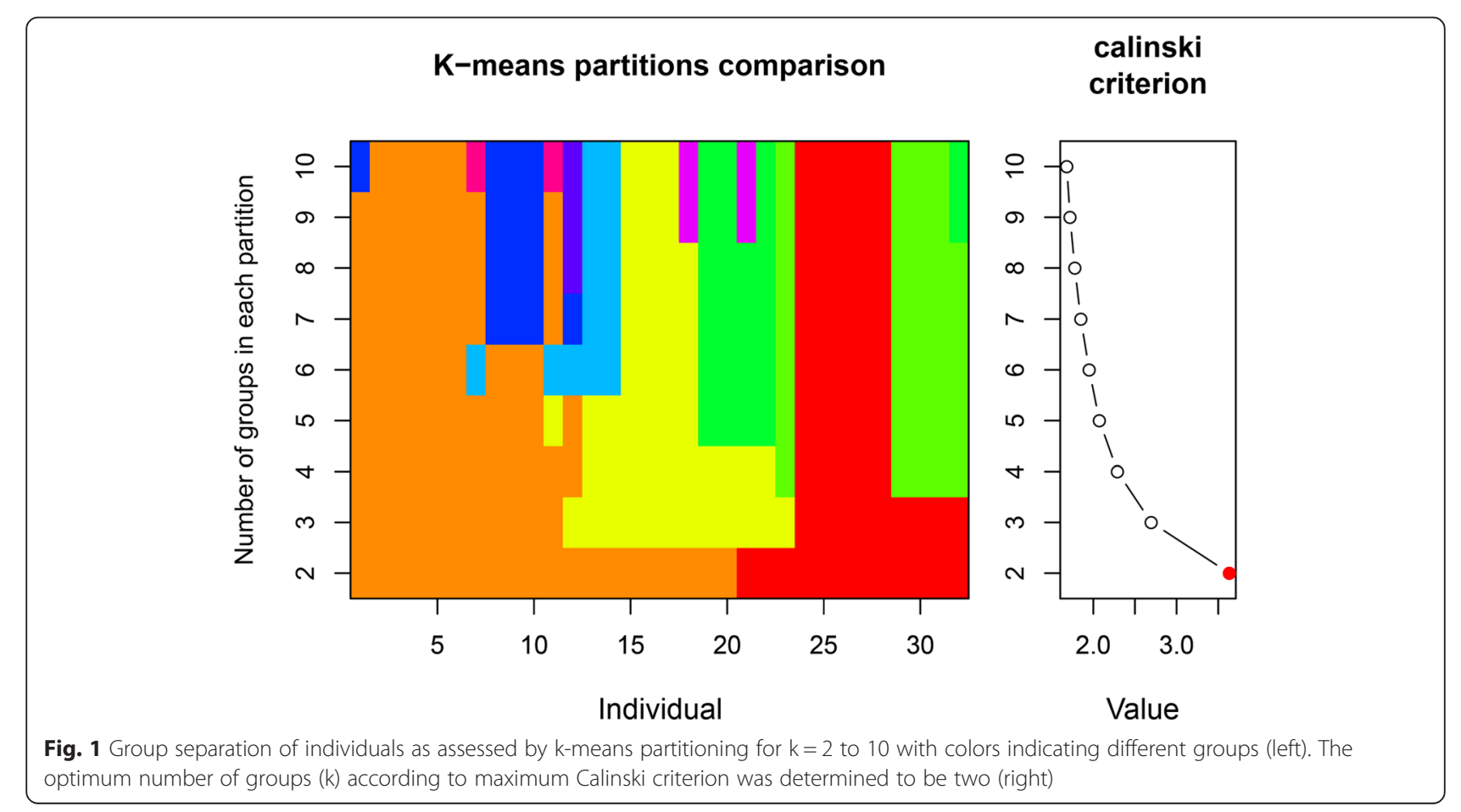


simulate a grouping of individuals (assigned by different colors) dependent on number of groups chosen. Individuals were assigned into two to ten groups, with a more homogenous grouping for two and three groups. The Calinski criterion (Fig. 1, right), giving the most likely grouping by the highest value reached, indicating a grouping of individuals into two groups by a value $>3$.

The cluster dendrogram based on the modified Roger's distance (Fig. 2) also displayed a partitioning of individuals in two main groups, which were separated by a modified Roger's distance value of 0.47 . Individuals belonging to the same variety located in the same main branch for the varieties Perly (ID_14, ID_18; 0.4), Visnovsky (ID_13, ID_28; 0.39) and Zeus (ID_29, ID_30; $0.48)$. The variety Perdix is an advanced variety originating from the variety Perly and the Perdix genotype (ID_16) clusters closely to one of the Perly individuals (ID_14).

The first, smaller branch of the cluster (Fig. 2, right hand side) consisted mainly of individuals originating from Switzerland and the United Kingdom (cluster 1), whereas the majority of the second, larger branch was comprised of individuals from Southern and Eastern Europe as well as individuals from USA, Morocco and Canada (cluster 2). However, AU values showed no significance (values <95) for most branches. Principal component analysis (PCA; Fig. 3) showed a pattern comparable to that observed from cluster analysis with individuals of the two main clusters mainly being separated by the first principle component which explained $10.3 \%$ of the total marker variation. The second principle component accounted for $4.9 \%$ of the variation, most of which was intragroup. The occurrence of alleles across all markers varied between the two clusters with 849 alleles amplified in cluster 1 (65.3 per individual) and 979 alleles in cluster 2 (51.5 per individual).

\section{Discussion}

The 101 SSR markers newly developed from sainfoin revealed a high degree of polymorphism. In addition to differences in multiples of the repeat motif, we also found alleles differing by fractions of the multiple motif length. Such variations could have arisen from insertions, deletions and translocations in the flanking region of the SSR [35]. Such mutations in the flanking region might also contribute to the high degree of polymorphism in our marker data set. The SSR sizes predicted through sequencing and the actual size distribution observed in the 32 individuals was consistent for most of the markers. Discrepancies can largely be explained by the fact that SSR motifs were developed from individuals not represented in the present study. In total, we found 1154 alleles at 101 loci resulting in 11.4 amplified alleles per SSR on average. This is twice the amount found by Demdoum [12], who found 5.83 alleles by transferring markers from barrel clover (Medicago truncatula Gaertn.) and soybean (Glycine max L.) to sainfoin. Fragments were smaller for the specific marker set in this study (92 to 511 bp) compared to markers adopted from other species [12] (79 to $865 \mathrm{bp}$ ). The larger sizes of alleles from crossspecies amplification could be attributed to interspecific differences to the donor species due to repeat length variation within the SSR region and indels in the flanking region [36]. Avci [37] amplified 725 alleles from 18 SSR markers in diverse Onobrychis spp. using markers from pea and barrel clover. The higher

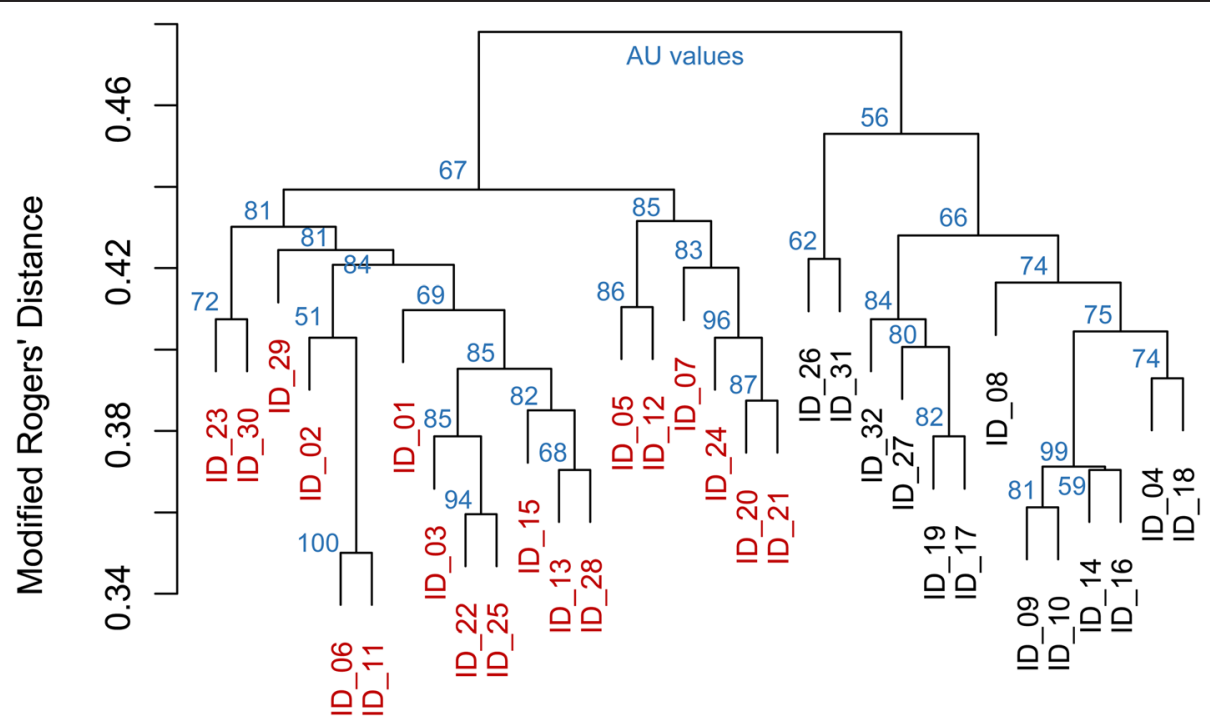

Fig. 2 Cluster Dendrogram of individuals based on the modified Rogers' distance. Values at branches are AU p-values (blue). Different colours of genotype labels give the affiliation to the two groups determined by k-means partitioning 


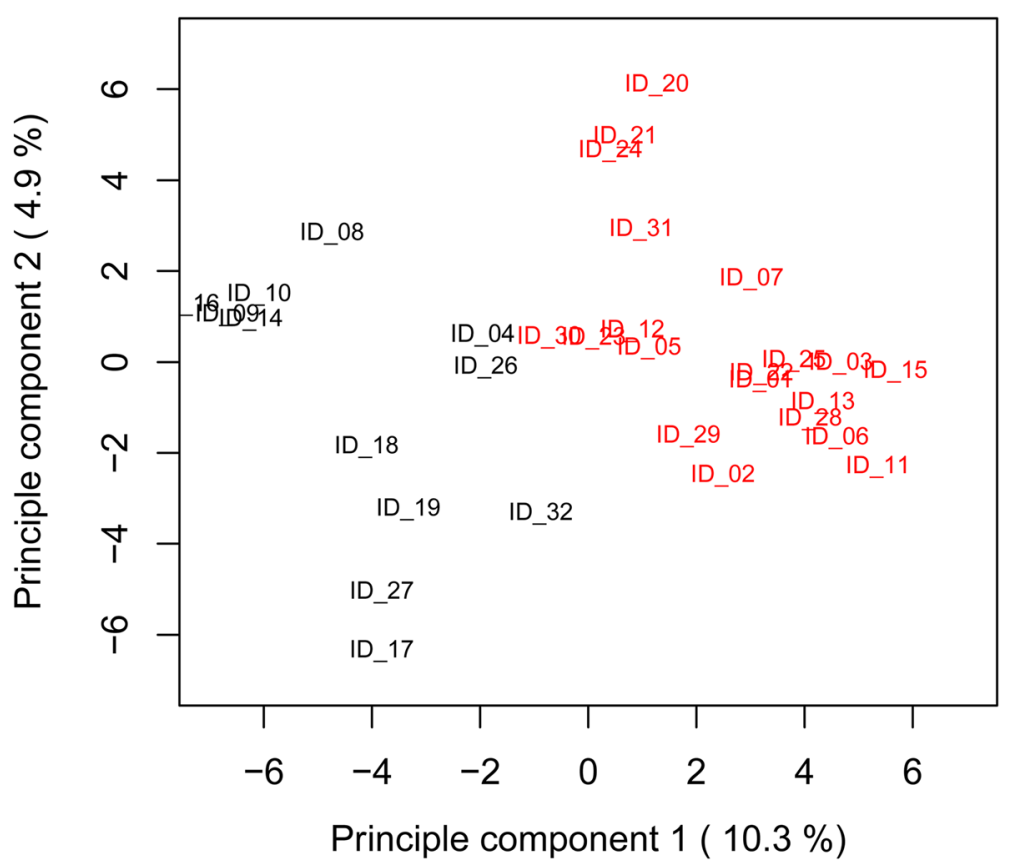

Fig. 3 Principle component analysis of 32 sainfoin (O. viciifolia) individuals based on 1054 alleles of 101 SSR markers. Different colors give the affiliation to the two groups as determined by k-means partitioning

number found by these authors could be explained by the larger diversity of germplasm used, which originated from different subspecies.

SSR marker studies with other tetraploid species using diverse panels of individuals showed lower numbers of alleles per marker compared to the present study, e.g. 7.2 alleles in sugar cane (Saccharum officinarum) [38], 6.7 alleles in switchgrass (Panicum virgatum) [39] and 6 alleles in peanut (Arachis hypogaea) [40].

A few markers were observed with less than five alleles among the 32 individuals. These may still be useful in future studies, since this study represents an initial screening of single individuals and not an extensive population survey. Additionally, using only the most polymorphic markers would bias the overall genetic diversity e.g. in conservation studies [41].

The challenge in analyzing SSR alleles in tetraploids lies in determining the dosage of each allele, which is often impossible using capillary electrophoresis for individuals carrying less than four different alleles at a specific marker locus. The PIC content gives an estimation of the information content of a marker and is traditionally calculated by the formula of Botstein [42]. This was developed for diploid species, for which the allele frequency is either known or can be inferred from the allele occurrence (presence/absence). For tetraploid species, the allele frequency is difficult to derive from the allele occurrence due to different allele doses (1 to 4 alleles). Hence, the formula for diploids could not be used for tetraploid sainfoin. Thus, the PIC was calculated separately for each allele, on the basis of allele occurrence counts, using a formula adopted from Roldan-Ruiz [26] and averaging the PIC across all loci of one locus [43]. Here, the maximum value that can be reached is 0.5 , which corresponds to alleles found in $50 \%$ of the population. Small values, on the other hand, correspond to very abundant or to very rare alleles. Deciding whether a SSR marker is useful also depends upon the scientific issue. Taking into account different allele-based PIC values of an SSR locus (Additional file 2: Figure S2), therefore, gives the most holistic picture of the SSR marker. High PIC values of alleles (0.5-0.4) are useful for inside population studies e.g. to trace marker trait associations, whereas low PIC values (0.0-0.1) of single alleles could be more useful for studies of evolution or genetic drift [44]. The average PIC values in this study indicated that most markers had alleles which could be found in a group of individuals and are suitable for several approaches in future studies. These PIC values were comparable to those found by Tehrani [43] which were between 0.16 and 0.44 in Lolium persicum Boiss. The large number of private alleles is a clear indication of genetic distinctness of the individuals, which was anticipated in view of their diverse origins.

Genetic diversity is a prerequisite for selection in variety development. So far, there is limited information on the genetic diversity of sainfoin available. Use of AFLP and SSR markers from other species were not able to 
reveal genetic diversity in distinct Spanish sainfoin accessions $[12,45]$. The values of that study, given by Nei's similarity values, which represent the proportion of shared fragments on the basis of binary data and corrected by the marker number [46], reached values of 0.73 to $0.8[12,45]$. A conversion of those values to genetic distance values by the formula - ln (Nei's similarity values) resulted in Nei's genetic distance values of 0.31 and 0.22 [47]. In a study of sainfoin genetic diversity using RAPD markers in ten landraces from East Azerbaijan and in 36 Iranian sainfoin populations, Nei's genetic distance values of 0.32 and 0.25 , respectively, were observed $[48,49]$. In our study, highest modified Roger's distance of 0.48 corresponds to alleles not shared between our two cluster groups, which is almost $50 \%$ (Fig. 2). The smallest Roger's distance values with 0.35 , corresponds to an approximate Nei's distance value of 0.43 (Additional file 1: Figure S1), which is higher than the low values observed in other studies [12, 45, 48]. The majority of among-genotype comparisons showed higher values. The higher values of genetic diversity found in the present study may reflect the high variability of the markers developed and the selection of 32 individuals of contrasting origin. Despite the fact that individuals of the same cultivars in this predominantly outbreeding species can show considerable variability [50], the individuals from the same cultivar grouped clearly together in the present study (Figs. 2 and 3, Table 1).

The 32 individuals investigated separated into two clear groups based on different multivariate analyses. The first main group was comprised mainly of individuals from Switzerland and the United Kingdom, whereas the second group contained individuals originating from South and East Europe as well as USA, Canada and Morocco. In some instances, individuals originating from the same geographical region did not cluster tightly together, some even into the two different cluster groups. The three plants from Italy, ID_29 and ID_30, both cultivar "Zeus", clustered in group 2, whereas ID_31 of the cultivar "Ambra" clustered to group 1). Especially for cultivars, this is likely to be due to different origin of base material (which is often unknown), as well as divergent breeding and selection history.

A similar grouping of accessions identified by the present cluster analysis could be found in earlier studies between sainfoin accessions from Western Europe and those from Eastern Europe and Asia [12, 23]. This clear genetic distinction between the individuals from Western Europe and those from Eastern Europe and beyond could reflect adaptation to diverse climatic conditions either naturally or as a result of local selection by growers [44]. Under genetic isolation and limited gene exchange, differentiation in the sainfoin germplasm with accompanied morphological separation seems likely [51]. The average number of alleles amplified in individuals of the West European cluster was 65.3, which was approximately 14 alleles more than individuals from the other cluster (51.5). These results might indicate a higher allelic diversity in individuals from mainly Switzerland and Great Britain compared to other origins. Deducing differences in tannin content and composition between single individuals of the two clusters based on earlier studies dealing with samples of plants from the same accessions is extremely difficult because the variation found within accessions is at least as large as variation between accessions [24].

\section{Conclusions}

This study reports the first characterization of specific co-dominant SSR markers for sainfoin. The 101 SSR markers characterized in this study showed a high degree of polymorphism and clearly demonstrated the differences between sainfoin individuals, with diverse origin, on a molecular genetic level. The genetic differences found in our panel separated the individuals into two groups, with a clear correlation to the geographical origin of those individuals. SSR markers, such as those characterized here, will be very useful in future genetic analyses, such as paternity or pedigree analysis in breeding programs, as well as more detailed analysis of genetic diversity in this forage crop. Furthermore, the development of new varieties could be crucially improved by choosing distinct genepools and minimising inbreeding depression.

\section{Additional files}

Additional file 1: Figure S1. Relationship between modified Roger's Distance to Euclidian Distance and to Nei's Distance. (PDF 319 kb)

Additional file 2: Figure S2. Polymorphism Information Content (PIC) values for individual alleles at SSR loci. Different grey levels are used for better visual differentiation among alleles of the different SSR markers. (PDF $431 \mathrm{~kb}$ )

\section{Acknowledgements}

We wish to thank the European Commission for funding this research (Marie Curie Initial Training Network, 'LegumePlus', PITN-GA-2011-289377). The authors are grateful to Rhys Kelly and Kirsten Skøt (IBERS) and Tom Wood (NIAB) for help with the SSR analysis.

\section{Funding}

This research was funded by the European Commission through a Marie Curie Initial Training Network, 'LegumePlus', PITN-GA-2011-289377.

\section{Availability of data and materials}

The list of primers and accessions used are provided in the paper. The full list of all putative SSR loci and the sainfoin transcriptome sequence is provided in a manuscript currently under review, and is also available from the corresponding author.

\section{Authors' contributions}

$\mathrm{KK}$ and $\mathrm{MMO}$ contributed to the experimental design, performed all analyses and led the writing of the manuscript. LMJS assisted in data collection, 
variety choice, structuring the work programme and helped to draft the manuscript. RK and LS conceived the study and assisted with data collection, data analysis and drafting the manuscript. All authors read and approved the final version of the manuscript.

\section{Competing interests}

The authors declare that they have no competing interests.

\section{Consent for publication}

Not applicable.

\section{Ethics approval and consent to participate}

No ethics approval were needed for this work.

\section{Author details}

Agroscope ISS, Reckenholzstrasse 191, CH-8046 Zürich, Switzerland

${ }^{2}$ National Institute of Agricutural Botany, Huntingdon Road, Cambridge CB3

OLE, UK. ${ }^{3}$ IBERS, Aberystwyth University, Gogerddan, Aberystwyth, Ceredigion SY23 3EB, UK.

Received: 13 April 2016 Accepted: 22 August 2016

Published online: 30 August 2016

\section{References}

1. Koivisto JM, Lane GPF. Sainfoin - worth another look. In: College RA, editor. Sainfoin: worth another look. Royal Agricultural College, Cirencester, on behalf of the BGS Forage Legumes Special Interest Group, UK. Cirencester; 2001.

2. Burton JC, Curley RL. Nodulation and nitrogen fixation in sainfoin (Onobrychis sativa, Lam.) as influenced by strains of rhizobia. Mont AES Bull. 1970;627:3-5.

3. Frame J, Charlton JFL, Laidlaw AS. Sainfoin. In: International C, editor. Temperate forage legumes. Wallingford: CAB International; 1998. p. 279-89.

4. Sheehy JE, Popple SC. Photoynthesis, water relations, temperature and canopy structure as factors influencing the growth of sainfoin (Onobrychis viciifolia Scop.) and lucerne (Medicago sativa L.). Ann Bot. 1981:48:113-28.

5. Hoste H, Jackson F, Athanasiadou S, Thamsborg SM, Hoskin SO. The effects of tannin-rich plants on parasitic nematodes in ruminants. Trends Parasitol. 2006;22:253-61.

6. Min BR, Barry TN, Attwood GT, McNabb WC. The effect of condensed tannins on the nutrition and health of ruminants fed fresh temperate forages: a review. Anim Feed Sci Technol. 2003;106:3-19.

7. Mueller-Harvey I. Unravelling the conundrum of tannins in animal nutrition and health. J Sci Food Agric. 2006;86:2010-37.

8. Marais JPJ, Mueller-Harvey I, Brandt EV, Ferreira D. Polyphenols, condensed tannins, and other natural products in Onobrychis viciifolia (sainfoin). J Agric Food Chem. 2000:48:3440-7.

9. Ramirez-Restrepo CA, Barry TN. Alternative temperate forages containing secondary compounds for improving sustainable productivity in grazing ruminants. Anim Feed Sci Technol. 2005;120:179-201.

10. Goplen BP, Richards KW, Moyer JR. Sainfoin for western Canada. Agriculture Canada Publication. 1991;1470E:24.

11. Zhang T, Wang $Y$, Wang $Q$, Wang $X$, Han J. Organic carbon and nitrogen stocks in reed meadow soils converted to alfalfa fields. Soil Till Res. 2009:105:143-8.

12. Demdoum S, Munoz F, Delgado I, Valderrabano J, Wuensch A. EST-SSR cross-amplification and genetic similarity in Onobrychis genus. Genet Resour Crop Evol. 2012;59:253-60.

13. Lewke Bandara N, Papini A, Mosti S, Brown T, Smith LMJ. A phylogenetic analysis of genus Onobrychis and its relationships within the tribe Hedysareae (Fabaceae). Turk J Bot. 2013:37:981-92

14. Hayot-Carbonero C. Sainfoin (Onobrychis viciifolia), a forage legume with great potential for sustainable agriculture, an insight on its morphological, agronomical, cytological and genetic characterisation. In: PhD Thesis. Manchester: University of Manchester; 2011.

15. Kempf K, Grieder G, Walter A, Widmer F, Reinhard S, Koelliker R. Evidence and consequences of selffertilisation in the predominantly outbreeding forage legume Onobrychis viciifolia. BMC Genet. 2015;16:1-12.

16. Sorrells ME, Wilson WA. Direct classification and selection of superior alleles for crop improvement. Crop Sci. 1997:37:691-7.

17. Litt M, Luty JA. A hypervariable microsatellite revealed by in vitro amplification of a dinucleotide repeat within the cardiac muscle actin gene. Am J Hum Genet. 1989;44:397-401.
18. Powell W, Machray GC, Provan J. Polymorphism revealed by simple sequence repeats. Trends Plant Sci. 1996;1:215-22.

19. Kumar P, Gupta VK, Misra AK, Modi DR, Pandey BK. Potential of Molecular Markers in Plant Biotechnology. Plant Omics. 2009;2:141-62.

20. Morgante $M$, Hanafey $M$, Powell W. Microsatellites are preferentially associated with nonrepetitive DNA in plant genomes. Nat Genet. 2002;30: 194-200.

21. Smith DN, Devey ME. Occurrence and inheritance of microsatellites in Pinus radiata. Genome. 1994;37:977-83.

22. Chen H, Liu L, Wang L, Wang S, Somta P, Cheng X. Development and Validation of EST-SSR Markers from the Transcriptome of Adzuki Bean (Vigna angularis). PLoS One. 2015;10:e0131939. Article No.: e0131939.

23. Hayot-Carbonero CH, Carbonero F, Smith LMJ, Brown TA. Phylogenetic characterisation of Onobrychis species with special focus on the forage crop Onobrychis viciifolia Scop. Genet Resour Crop Evol. 2012;59:1777-88.

24. Malisch CS, Luescher A, Baert N, Engstrom MT, Studer B, Fryganas C, Suter D, Mueller-Harvey I, Salminen J-P. Large Variability of Proanthocyanidin Content and Composition in Sainfoin (Onobrychis viciifolia). J Agric Food Chem. 2015:63:10234-42

25. Schuelke M. An economic method for the fluorescent labeling of PCR fragments. Nat Biotechnol. 2000:18:233-4.

26. Roldan-Ruiz I, Dendauw J, Van Bockstaele E, Depicker A, De Loose M. AFLP markers reveal high polymorphic rates in ryegrasses (Lolium spp.). Mol Breed. 2000;6:125-34.

27. Wright S. Variability within and among natural populations, vol. 4. Chicago: The University of Chicago Press; 1978.

28. Lee M, Godshalk EB, Lamkey KR, Woodman WW. Association of restriction fragment length polymorphisms among maize inbreds with agronomic performance of their crosses. Crop Sci. 1989;29:1067-71.

29. Suzuki R, Shimodaira H. Pvclust: an R package for assessing the uncertainty in hierarchical clustering. Bioinformatics. 2006;22:1540-2.

30. Shimodaira $\mathrm{H}$. An approximately unbiased test of phylogenetic tree selection. Syst Biol. 2002;51:492-508.

31. Shimodaira H. Approximately unbiased tests of regions using multistepmultiscale bootstrap resampling. Ann Stat. 2004;32:2616-41.

32. Efron B. Bootstrap methods: another look at the jackknife. Ann Stat. 1979:7:1-26.

33. MacQueen J. Some methods for classification and analysis of multivariate observations. In: Fifth Berkeley Symposium on Mathematical Statistics and Probability. Berkeley: University of California Press; 1967.

34. Calinski T, Harabasz J. A dendrite method for cluster analysis. Comm Stat. 1974;3:1-27.

35. Robinson JD, Hall DW, Wares JP. Approximate Bayesian estimation of extinction rate in the Finnish Daphnia magna metapopulation. Mol Ecol. 2013;22:2627-39.

36. Peakall R, Gilmore S, Keys W, Morgante M, Rafalski A. Cross-species amplification of soybean (Glycine max) simple sequence repeats (SSRs) within the genus and other legume genera: Implications for the transferability of SSRs in plants. Mol Biol Evol. 1998;15:1275-87.

37. Avci S, Ilhan E, Erayman M, Sancak C. Analysis of Onobrychis genetic diversity using SSR markers from related legume species. J Anim Plant Sci. 2014:24:556-66

38. Pinto LR, Oliveira KM, Marconi T, Garcia AAF, Ulian EC, de Souza AP. Characterization of novel sugarcane expressed sequence tag microsatellites and their comparison with genomic SSRs. Plant Breeding. 2006;125:378-84.

39. Narasimhamoorthy B, Saha MC, Swaller T, Bouton JH. Genetic Diversity in Switchgrass Collections Assessed by EST-SSR Markers. Bioenerg Res. 2008;1:136-46.

40. Hopkins MS, Casa AM, Wang T, Mitchell SE, Dean RE, Kochert GD, Kresovich S. Discovery and characterization of polymorphic simple sequence repeats (SSRs) in peanut. Crop Sci. 1999;39:1243-7.

41. Vali U, Einarsson A, Waits L, Ellegren $H$. To what extent do microsatellite markers reflect genome-wide genetic diversity in natural populations? Mol Ecol. 2008;17:3808-17

42. Botstein D, White RL, Skolnick M, Davis RW. Construction of a genetic linkage map in man using restriction fragment length polymorphisms. Am J Hum Genet. 1980;32:314-31.

43. Tehrani MS, Mardi M, Saeidi H, Gharehyazi B, Assadi M. Transferability of genomic and EST-microsatellites from Festuca arundinacea Schreb. to Lolium persicum Boiss. and Hohen. ex Boiss. Int J Bot. 2008;4:476-80.

44. Silvertown JW, Doust JL. Introduction to plant population biology. 3rd ed. Oxford: Blackwell Scientific Publications LTD; 1993. 
45. Sardaro S, Molinari L, Albertini E, Reosellini D, Negri V, Falcinelli M. Distinguibilità molecolare di poplazioni spontanee di Poa pratensis, Lolium perenne e Onobrychis viciifolia. Sementi Elette. 2003;6:47-9.

46. Lamboy WF. Computing genetic similarity coefficients from RAPD data: the effects of PCR artifacts. PCR Meth Appl. 1994;4:31-7.

47. Frankham R, Ballou J, Briscoe DA. Introduction to conservation genetics. Cambridge: Cambridge University Press; 2010.

48. Hejrankesh N, Mousavizadeh SA, Haghighi AR, Rashidi V. Evaluation of genetic diversity of sainfoin (Onobrychis vicifolia Scop.) landraces using RAPD markers. J Curr Res Sci. 2014;2:739-48.

49. Rasouli M, Jafari AA, Tabaei-Aghdaei SR, Shanjani PS, Darvish F. Assessment of genetic variability of 36 populations of sainfoin (Onobrychis sativa) based on RAPD markers. Int J Biosci. 2013:3:15-26.

50. Posselt UK. Breeding Methods in Cross-Pollinated Species. In: Boller B, Posselt UK, Veronesi F, editors. Fodder Crops and Amenity Grasses. New York: Springer; 2010.

51. Zarrabian M, Majidi MM, Ehtemam MH. Genetic Diversity in a Worldwide Collection of Sainfoin Using Morphological, Anatomical, and Molecular Markers. Crop Sci. 2013;53:2483-96.

\section{Submit your next manuscript to BioMed Central} and we will help you at every step:

- We accept pre-submission inquiries

- Our selector tool helps you to find the most relevant journal

- We provide round the clock customer support

- Convenient online submission

- Thorough peer review

- Inclusion in PubMed and all major indexing services

- Maximum visibility for your research

Submit your manuscript at www.biomedcentral.com/submit 